\title{
BMJ Open Treatment goal setting for complex patients: protocol for a scoping review
}

\author{
Agnes Grudniewicz, ${ }^{1,2}$ Michelle Nelson, ${ }^{1}$ Kerry Kuluski, ${ }^{1,2}$ Vincci Lui, ${ }^{3}$ \\ Heather V Cunningham, ${ }^{3}$ Jason X Nie, ${ }^{1}$ Heather Colquhoun, ${ }^{4}$ Walter $\mathrm{P}$ Wodchis, ${ }^{2}$ \\ Susan Taylor, ${ }^{5}$ Mayura Loganathan, ${ }^{6}$ Ross E Upshur ${ }^{1,7}$
}

To cite: Grudniewicz A, Nelson M, Kuluski K, et al. Treatment goal setting for complex patients: protocol for a scoping review. BMJ Open 2016;6:e011869. doi:10.1136/bmjopen-2016011869

- Prepublication history and additional material is available. To view please visit the journal (http://dx.doi.org/ 10.1136/bmjopen-2016011869).

Received 11 March 2016 Revised 19 April 2016 Accepted 5 May 2016

\section{CrossMark}

For numbered affiliations see end of article.

\footnotetext{
Correspondence to Dr Agnes Grudniewicz; a.grudniewicz@mail.utoronto. ca
}

\section{ABSTRACT}

Introduction: An increasing number of people are living longer with multiple health and social care needs, and may rely heavily on health system resources. When dealing with multiple conditions, patients, caregivers and healthcare providers (HCPs) often experience high treatment burden due to unclear care trajectories, a myriad of treatment decisions and few guidelines on how to manage care needs. By understanding patient and caregiver priorities, and setting treatment goals, HCPs may help improve patient outcomes and experiences. This study aims to examine the extent and nature of the literature on treatment goal setting in complex patients, identify gaps in evidence and areas for further inquiry and guide a research programme to develop definitions, measures and recommendations for treatment goal setting.

Methods and analysis: This study protocol outlines a scoping review of the peer reviewed and the grey literature, using established scoping review methodology. Literature will be identified using a multidatabase and grey literature search strategy developed by two librarians. Papers and reports on the topic of goal setting that address complexity or complex patients will be included. Results of the search will be screened independently by two reviewers and included studies will be abstracted and charted in duplicate.

Ethics and dissemination: Ethics approval is not required for this scoping review. Working with the knowledge users on the team, we will prepare educational materials and presentations to disseminate study findings to HCPs, caregivers and patients, and at relevant national and international conferences. Results will also be published in a peer-reviewed journal.

\section{INTRODUCTION}

An increasing number of people in the industrialised world are living longer, often with multiple health and social care needs. ${ }^{1}$ When dealing with multiple conditions, these patients, their caregivers and their healthcare providers (HCPs) often experience high treatment burden and/or burnout from unclear care trajectories, countless treatment decisions and few guidelines on

\section{Strengths and limitations of this study}

- Strengths of this study include the importance of the topic to patient care, use of established scoping review methodology, a systematic search developed by two health sciences librarians and systematic screening and data abstraction carried out in duplicate.

- A limitation of this review is the potential to miss relevant articles, especially in the grey literature. To mitigate this, we will consult with experts in the field of patient complexity to attempt to identify any missed articles and scan reference lists of included articles and similar reviews.

how to manage care needs. Adults with more than one chronic condition are the largest users of healthcare services and account for more than $60 \%$ of healthcare spending. ${ }^{2}{ }^{3}$ While a considerable proportion of people manage their chronic conditions with minimal healthcare needs, others are considered complex due to factors such as multimorbidity, high service use and psychosocial vulnerability. ${ }^{45}$

Complex patients experience challenges that can span across multiple health dimensions (medical/physical health, mental health, patient demographics, social capital and health and social experiences), ${ }^{4}$ and it is the intersection and interaction across these dimensions that are inherently complex. There is no agreed on definition or minimum number of co-occurring conditions required to reach complexity; some single conditions (eg, dementia and stroke) can be complex in and of themselves. ${ }^{6}{ }^{7}$ For the purpose of this review, we have defined complexity as, at minimum, more than one concurrent chronic condition, compounded by frailty, palliative or life-limiting serious illness diagnoses, mental health conditions and/or social challenges, or the presence of a complex chronic illness (eg, dementia and stroke) that spans across more than one health dimension (as presented in the Complexity Framework). ${ }^{4}$ 
Managing complex patients in healthcare systems organised mainly by medical condition is challenging. As complexity increases, so does the number of HCPs involved in a patient's care. ${ }^{245}$ Furthermore, treatments for each disease can negatively impact each other, ${ }^{8}$ and clinical practice guidelines rarely provide guidance for multimorbidity and complexity. ${ }^{9-11}$ As such, primary care physicians have expressed concerns about managing care and making decisions about treatments, regimen manageability, and corresponding risks and benefits for complex patients. ${ }^{4}$ Aligning patient goals with HCP care goals may improve outcomes in this patient population by prioritising treatments to meet patient preferences amid the many and often competing medical recommendations. ${ }^{12}$

Little is known about the process clinicians use to set goals in the presence of complexity and we lack evidence to support best practices in goal setting for complex patients. To the best of our knowledge, there is no comprehensive review to guide the implementation or evaluation of goal setting interventions for complex patients. A related stream of research exists on shared decision-making - a broader and complementary patient-centred intervention that can include the elicitation of patient goals. ${ }^{13}{ }^{14}$ However, in a review of shared decision-making, only $67 \%$ of studies identified 'patient values/preferences' as a component of the shared decision-making process. This points to the potential lack of attention to patient treatment goals, warranting a separate, in-depth study on the topic. ${ }^{14} \mathrm{~A}$ recent Cochrane review found that care planning (including goal setting) for adults with single chronic conditions led to improvements in some health outcomes, but the review did not explore the more challenging process of setting goals when patients experience complexity. ${ }^{15}$ Another Cochrane review of goal setting for adults with acquired disability found some low quality evidence for the effectiveness of goal setting, but explored neither complexity nor comorbidity. ${ }^{16}$ Berntsen $e t a l^{17}$ also identified a gap in research in their thematic analysis of goal concepts in the health services literature, stating that goal setting practices have not been explored. Furthermore, in a recent qualitative study, Kuluski et $a l^{12}$ found little goal alignment across triads of complex patients, caregivers and physicians, identifying the need for research on how to embed goal setting and alignment as a standard of practice.

This paper outlines the protocol for a scoping review that will methodically examine the extent and nature of the literature on treatment goal setting that is applicable to complex patients. We will explore HCPs setting goals with complex patients, collaborative goal setting (between HCPs, patients and/or caregivers) and patient-led goal setting within healthcare settings. The objectives of this review are to produce the first synthesis of the peer reviewed and the grey literature on goal setting for complex patients, identify gaps in evidence and areas for further inquiry, and guide a research programme to develop definitions, measures and recommendations for treatment goal setting.

\section{METHODS}

We will conduct a scoping review to examine the literature on treatment goal setting that is applicable to complex patients. This synthesis method will allow us to explore the broad topic of goal setting and make use of knowledge from across study designs and in both the peer reviewed and the grey literature. The scoping review is a sound method for mapping areas of research and presenting results in an accessible format for knowledge users. Though it does not entail quality assessment, it is a rigorous and systematic approach to knowledge synthesis.

We will follow the scoping review methods outlined by Arksey and O'Malley, ${ }^{18}$ and advanced by Levac et al. ${ }^{19}$ Arksey and O'Malley propose a six step framework for conducting scoping review studies, including: (1) identifying the research question, (2) identifying relevant literature, (3) study selection, (4) charting the data, (5) collating, summarising and reporting the articles and (6) consulting and translating knowledge. ${ }^{18}$ Our study team includes knowledge users (ST and ML) to ensure relevance of the study to patient care and to facilitate dissemination of study results.

\section{Identification of the relevant literature \\ Search strategy}

The comprehensive search strategy was developed by two health sciences librarians (VL and HVC). The search strategy was validated through the retrieval of a key set of relevant studies, and identified 8935 citations when executed in MEDLINE (see online supplementary appendix 1 for MEDLINE search). The patient complexity terms were informed by the authors' definition of patient complexity, and partly based on a scoping review of patient complexity, ${ }^{4}$ and include: comorbidity, multiple comorbidity, multimorbidity, polypathology and pluripathology. The search terms for the concept of goal setting, including goal setting, goal alignment, as well as target, preference and priority setting, were adapted from a Cochrane review on goal setting in rehabilitation for disabilities. ${ }^{16}$ The MEDLINE search strategy will be translated in EMBASE (1947-current), PsycINFO (1806-current), CINAHL (1981-current), AMED (1985-current), AgeLine (1978-current), Scopus (1970-current) and the Cochrane Library. Reference lists of included studies will be searched for citations.

We will also conduct a thorough search of the grey literature to identify any non-indexed literature, including dissertation abstracts, government documents, conference proceedings, practice guidelines, clinical aids, educational materials and reports, through searching sources such as Open Grey, Conference Proceedings Index, ProQuest Dissertations and Theses, as well as Google. Experts in the field of patient complexity will be 
contacted and consulted in order to facilitate identification of relevant published, in progress, or unpublished research data. All literature searches will be conducted by the two experienced librarians (VL and HVC) on the study team. Searches will be limited to the English language, without date or study type restrictions. Bibliographic information of included articles will be amalgamated and stored using the citation management programme, EndNote.

\section{Study selection}

Titles and abstracts will be independently reviewed against the selection criteria and will be marked as 'include', 'exclude' or 'uncertain', by two reviewers; discrepancies will be resolved by discussion and consensus between the two reviewers, and with a third party in case no resolution can be attained. Full text articles will be retrieved for studies deemed as 'included' or 'uncertain', and will be reviewed independently and in duplicate for inclusion against the selection criteria. All reviewers will use a pilot-tested screening form developed for this review, at each stage of screening (see online supplementary appendix 2).

We will include published and unpublished literature reporting any quantitative, qualitative, mixed or multimethods research, including both comparative (eg, randomised, controlled, cohort, quasi-experimental) and non-comparative (eg, survey, narrative, audit) methods, educational materials and reports related to the topic of treatment goal setting for complex patients. We will include all goal setting papers that meet any of the following criteria, they: (1) directly use the term 'complexity', (2) address more than one concurrent chronic condition, compounded by frailty, palliative or lifelimiting serious illness diagnoses, mental health conditions and/or social challenges or (3) focus on a complex chronic illness (eg, dementia and stroke) that spans across more than one health dimension (as presented in the Complexity Framework). ${ }^{4}$ Given the challenging nature of defining and identifying patient complexity, studies marked 'uncertain' during full-text screening will be discussed among team members until a consensus is reached regarding inclusion. Studies focused on a single disease (eg, diabetes, heart failure) without consideration of complexity (as outlined above) will be excluded.

\section{Data extraction}

All included studies will be reviewed and charted independently by two reviewers, using a pilot-tested data abstraction form. Charting is a technique for organising and interpreting data by sifting, categorising and sorting material, according to key issues and themes. ${ }^{18}$ We will run the data abstraction pilot-test for $\sim 5$ articles to ensure consistency and to test the data abstraction form. Necessary changes will be made and shared with the team prior to abstracting the remaining articles. The following data will be extracted when available (but may change based on included studies): study citation, publication type (eg, published and unpublished), study type (eg, quantitative, qualitative and commentary/report), study characteristics (eg, study location, healthcare setting and use of control), patient characteristics (eg, number of patients, age and comorbidities), provider characteristics (eg, number of providers, age and specialty), involvement of caregiver (eg, yes/no and type of caregiver), intervention details (eg, length, process of goal setting, use of care coordination plan and health dimensions addressed), definition of complexity, outcome measures and outcomes/study results (eg, quantitative results, qualitative themes, recommendations, key learnings, goals set and degree of alignment). All study outcomes will be extracted, including patient, caregiver, HCP or system-level quantitative or qualitative (eg, satisfaction) outcomes.

\section{Synthesis and presentation of results}

In order to present an overview of all the information retrieved, and to establish the extent and nature of the literature, the results of the review will be presented using two strategies: (1) a basic numerical overview of the amount, type and distribution of included studies and (2) a narrative synthesis and mapping of the results. As an overview, we will create a table of included studies, listing for each (when appropriate and available): study type, aims, care settings, goal setting processes (if described), outcome measures and study outcomes (quantitative and qualitative). Though the exact reporting format cannot be established until data are charted and discussed with the knowledge users on the team, we will provide a narrative synthesis of included studies and use the Complexity Framework to support mapping of data. ${ }^{4}$ The framework provides a foundation within which to understand complexity, and outlines five health dimensions of complexity (health and social experiences, demographics, medical/physical health, social capital and mental health), which can be used to understand the extent and nature of the included studies, and the gaps and challenges identified in the literature. If appropriate, we will use deductive thematic analysis on relevant extracted study data (eg, goal setting process and definition of complexity) to map studies onto the health dimensions and demonstrate how complexity was considered in the literature. If possible, we will map the goal setting process, however, as per scoping review methods described by Arksey and O'Malley, ${ }^{18}$ and by Levac et $a l,{ }^{19}$ we anticipate the charting of the data will be an iterative process that depends on the literature found. Neither a study quality assessment nor a meta-analysis of quantitative results will be conducted as they are not a part of the objectives of the study or the scoping review methodology. ${ }^{18}$ All included studies will be synthesised.

\section{Knowledge translation and consultation}

We are employing an integrated knowledge translation approach by engaging knowledge users throughout all 
stages of the study. Our team includes two knowledge users, a primary care physician (ML) and a policymaker (ST), along with additional collaborators to ensure that study findings meet the needs of HCPs and policymakers, to offer their perspectives throughout the study, to suggest additional sources of information and to spearhead end-of-study knowledge translation. Consultation with knowledge users occurred during study design and will continue for discussion of preliminary findings, to validate results and to inform future research. ${ }^{19}$ Furthermore, we will share preliminary findings with HCPs outside the study team to validate results and support knowledge translation efforts. We will prepare educational materials and presentations to disseminate study findings to HCPs, caregivers and patients, and at relevant national and international conferences. Results will also be published in a peer-reviewed journal.

\section{CONCLUSION}

This paper presents the protocol for a systematic scoping review of both the peer reviewed and the grey literature on goal setting for complex patients. This review will advance knowledge on goal setting for complex patients, a topic gaining interest due to the significant treatment burden and lack of decision-making support experienced by complex patients, their caregivers and HCPs. ${ }^{2}$ By synthesising the literature on this potential solution to challenges in providing care in the presence of complexity, this research will inform a global movement of policies and reforms for theseoften high-cost-users of health and social systems. Our systematic search will identify theories, interventions for patients and providers, recommendations, best practices and approaches to eliciting, setting and aligning goals between patients, HCPs and/or caregivers. By identifying gaps in the literature for further research, this review will guide a research programme with the aim of developing definitions, measures and recommendations for treatment goal setting. If sufficient literature is found, a future systematic review and meta-analyses with a risk of bias assessment may be merited.

\author{
Author affiliations \\ ${ }^{1}$ Collaboratory for Research and Innovation, Lunenfeld-Tanenbaum Research \\ Institute, Sinai Health System, Toronto, Ontario, Canada \\ ${ }^{2}$ Institute of Health Policy, Management and Evaluation, University of Toronto, \\ Toronto, Ontario, Canada \\ ${ }^{3}$ Gerstein Science Information Centre, University of Toronto, Toronto, Ontario, \\ Canada \\ ${ }^{4}$ Department of Occupational Science and Occupational Therapy, University of \\ Toronto, Toronto, Ontario, Canada \\ ${ }^{5}$ Health Quality Ontario, Toronto, Ontario, Canada \\ ${ }^{6}$ Sinai Health System, Toronto, Ontario, Canada \\ ${ }^{7}$ Dalla Lana School of Public Health, University of Toronto, Toronto, Ontario, \\ Canada
}

Contributors AG and REU conceived the study. AG, HVC, REU, KK, MN, ML, ST and WPW contributed to the protocol design and plan. VL and HVC developed and conducted the literature search. All the authors worked collaboratively to draft and revise the manuscript, and read and approved the final version. All the authors made substantive intellectual contributions to the development of this protocol.

Funding Financial support was provided by the Health System Performance Research Network funded by the Ontario Ministry of Health and Long Term Care (Fund \#06034). The views expressed in this manuscript are those of the authors and do not necessarily represent the views of the funders.

\section{Competing interests None declared.}

Provenance and peer review Not commissioned; externally peer reviewed.

Open Access This is an Open Access article distributed in accordance with the Creative Commons Attribution Non Commercial (CC BY-NC 4.0) license, which permits others to distribute, remix, adapt, build upon this work noncommercially, and license their derivative works on different terms, provided the original work is properly cited and the use is non-commercial. See: http:// creativecommons.org/licenses/by-nc/4.0/

\section{REFERENCES}

1. Osborn R, Moulds D, Schneider EC, et al. Primary care physicians in ten countries report challenges caring for patients with complex health needs. Health Aff 2015;34:2104-12.

2. Osborn R, Moulds D, Squires D, et al. International survey of older adults finds shortcomings in access, coordination, and patient-centered care. Health Aff 2014;33:2247-55.

3. Tinetti ME, Fried TR, Boyd CM. Designing health care for the most common chronic condition-multimorbidity. JAMA 2012;20: 2493-4.

4. Schaink AK, Kuluski K, Lyons RF, et al. A scoping review and thematic classification of patient complexity: offering a unifying framework. J Comorbidity 2012;2:1-9.

5. Vogeli C, Shields AE, Lee TA, et al. Multiple chronic conditions: prevalence, health consequences, and implications for quality, care management, and costs. J Gen Intern Med 2007;22:391-5.

6. Robinson AL, Emden CG, Elder JA, et al. Multiple views reveal the complexity of dementia diagnosis. Australas J Ageing 2008;27:183-8.

7. Nelson M, Hanna E, Hall S, et al. What makes stroke rehabilitation patients complex? Clinician perspectives and the role of discharge pressure. J Comorbidity 2016;6:35-41.

8. Tsasis P, Bains J. Management of complex chronic disease: facing the challenges in the Canadian health-care system. Health Serv Manage Res 2008;21:228-35.

9. Upshur REG. Seven characteristics of medical evidence. J Eval Clin Pract 2000;6:93-7.

10. Fortin M, Contant E, Savard C, et al. Canadian guidelines for clinical practice: an analysis of their quality and relevance to the care of adults with comorbidity. BMC Fam Pract 2011;12:74.

11. Boyd CM, Darer J, Boult $\mathrm{C}$, et al. Clinical practice guidelines and quality of care for older patients. J Am Med Assoc 2005;294:716-24.

12. Kuluski K, Gill A, Naganathan G, et al. A qualitative descriptive study on the alignment of care goals between older persons with multi-morbidities, their family physicians and informal caregivers. BMC Fam Pract 2013;14:133.

13. Godolphin W. Shared decision-making. Healthc Q 2009;12:e186-90

14. Makoul G, Clayman ML. An integrative model of shared decision making in medical encounters. Patient Educ Couns 2006;60:301-12.

15. Coulter A, Entwistle Vikki A, Eccles A, et al. Personalised care planning for adults with chronic or long-term health conditions. Cochrane Database Syst Rev 2015;(3):CD010523.

16. Levack-William MM, Siegert RJ, Dean SG, et al. Goal setting and activities to enhance goal pursuit for adults with acquired disabilities participating in rehabilitation. Cochrane Database Syst Rev 2015;(7): CD009727.

17. Berntsen GKR, Gammon D, Steinsbekk A, et al. How do we deal with multiple goals for care within an individual patient trajectory? A document content analysis of health service research papers on goals for care. BMJ Open 2015;5:e009403.

18. Arksey H, O'Malley L. Scoping studies: towards a methodologica framework. Int J Soc Res Methodol 2005;8:19-32.

19. Levac D, Colquhoun H, O'Brien KK. Scoping studies: advancing the methodology. Implement Sci 2010;5:69. 